

\title{
Come posizionare i CVC: la venolisi chirurgica
}

\author{
A. Amato \\ Dipartimento Chirurgia, Ospedale di Sanremo, Sanremo (IM)
}

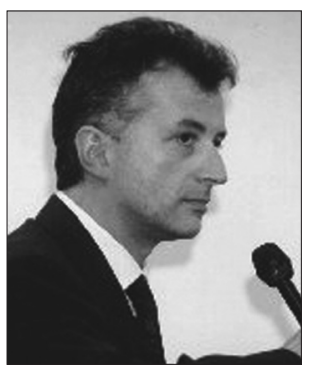

$\mathbf{F}$ ra le possibili scelte nell'identificare un accesso per un catetere venoso centrale in generale e di un sistema totalmente impiantabile (port) in particolare, l'isolamento chirurgico di una vena periferica rappresenta un'opzione tecnicamente semplice, eseguibile in anestesia locale, in regime ambulatoriale o di day surgery, con minimo discomfort per il paziente.

La vena giugulare esterna, unico ramo affluente della vena succlavia nella quale sbocca in prossimità della sua confluenza con la vena giugulare interna, rappresenta un accesso abitualmente agevole, prossimo a un piano osteomuscolare (a parete toracica anteriore) da utilizzare come struttura di ancoraggio del reservoir, in un distretto anatomico che consente un comodo accesso al port e non presenta articolazioni la cui escursione comporti il rischio di torsioni, angolazioni, dislocazioni o traumatismi del catetere.

La tecnica chirurgica prevede un'incisione di minima in sede sovraclaveare con isolamento della vena nell'atmosfera adiposa immediatamente sottostante il muscolo platisma. Con il paziente in posizione di Trendelmburg, al fine di ottenere un'adeguata replezione del vaso ed evitare il rischio di embolie gassose, si pratica una venotomia attraverso la quale fare procedere il catetere. Mediante controllo angiografico intra-operatorio, si verifica che l'apice del catetere sia posizionato in cava superiore. Da un punto di vista topografico è già possibile una valutazione sufficientemente attendibile, misurando la distanza fra il punto di inserzione del catetere e la seconda articolazione condrosternale (angolo del Luis), la quale corrisponde al termine del tronco brachiocefalico. Tuttavia il controllo radiologico è comunque indispensabile, per accertare che il catetere non abbia inavvertitamente preso direzioni errate (ad esempio v. succlavia controlaterale). La malposizione dell'apice del catetere è, infatti, una delle cause più frequenti di trombosi e formazione di guaina fibrosa con occlusione del lume. Il processo inizia con lesioni focali intimali e perdita dell'endotelio, e può proseguire con la proliferazione di cellule muscolari lisce a partenza dalla tonaca muscolare e ispessimento della parete venosa. Si generano, inoltre, adesioni focali fra catetere e parete venosa formate da trombi in vario stadio di organizzazione, collagene e cellule endoteliali.

Dopo aver confezionato una tasca sottocutanea in sede sottoclaveare omolaterale per l'impianto del reservoir, si tunnellizza il catetere e lo si raccorda al serbatoio. Occorre aver cura che lo spessore dei tessuti molli al di sopra del serbatoio sia intorno al centimetro: una distanza eccessiva fra cute e membrana del serbatoio potrebbe renderne problematico il reperimento e la puntura, mentre un lembo troppo sottile potrebbe esitare in ulcerazioni cutanee. Il sistema viene testato accertando il buon reflusso di sangue in aspirazione e la tenuta del raccordo catetere-serbatoio durante l'infusione. Il serbatoio viene fissato al piano muscolo-fasciale e al termine della procedura si esegue un lavaggio del sistema con soluzione fisiologica eparinata.

Il posizionamento del CVC a cielo aperto attraverso un vaso periferico consente di evitare le complicanze immediate più temibili, anche se rare, delle venipunture a cielo coperto, quali il pneumotorace, la puntura arteriosa accidentale con ematoma/emotorace, le lesioni del plesso brachiale, del dotto toracico (in caso di incannulamento a sinistra), del nervo ricorrente.

In alcuni casi, tuttavia, l'anatomia di questo distretto venoso può rendere problematico il corretto posizionamento del catetere. La vena giugulare esterna, infatti, nel suo tratto distale riceve alcuni affluenti quali la vena soprascapolare, la giugulare anteriore e la trasversa del collo. Nella maggior parte dei casi è presente anche un ramo anastomotico con la vena giugulare interna, ma numerose sono le varietà anatomiche che può presentare per origine, affluenti e terminazione.

Nel suo tratto terminale perfora quindi la fascia cervicale superficiale e media, 
e confluisce nella succlavia formando un angolo quasi retto aperto antero-medialmente. Questi dettagli anatomici rendono ragione delle difficoltà che talora si incontrano nella progressione del catetere e del fallimento di alcuni tentativi. Nella sua porzione distale, infine, presenta abitualmente due valvole, una negli ultimi $4 \mathrm{~cm}$ e una in corrispondenza del suo sbocco, fra le quali può dilatarsi un sinus. Questo ulteriore ostacolo può essere superato con l'artifizio di far progredire il catetere infondendo contemporaneamente soluzione fisiologica attraverso lo stesso, al fine di distendere la vena e dilatarne il lume.

Altri fattori possono addirittura rendere indaginoso il reperimento stesso della vena, quali pregressi interventi chirurgici del collo o la conformazione anatomica del paziente (obesità, brachitipia, collo taurino). Nei casi di fallimento, è possibile, attraverso lo stesso accesso chirurgico, isolare e incannulare la vena giugulare interna.

Analoghe rispetto alle altre tecniche di posizionamento sono invece le possibili complicanze tardive, quali l'occlusione del catetere, la reazione infiammatoria della tasca, la formazione della guaina fibrinica, l'infezione dell'impianto con eventuale sepsi, il dislocamento o la rottura/deconnessione del catetere, la trombosi, la necrosi cutanea. L'accesso giugulare, d'altro canto, è scevro dal rischio della cosiddetta pinch-off syndrome (sindrome del morso), che consiste nella progressiva erosione del catetere da parte delle strutture ossee, qualora il suo decorso avvenga a troppo stretto contatto dell'angolo sterno-claveare.

Occorre sottolineare, tuttavia, come le complicanze tardive siano talora il segno di una cattiva gestione del port: inosservanza delle norme dell'asepsi, utilizzo di aghi inadeguati (i normali aghi possono danneggiare la membrana e le particelle di silicone che si liberano ostruire il sistema) o di siringhe inadatte (siringhe di capacità inferiore a $10 \mathrm{~mL}$ possono generare pressioni troppo elevate nel catetere, fino a provocarne la rottura o il distacco), insufficiente manutenzione (il sistema va lavato dopo ogni utilizzo, e fra una somministrazione e l'altra in caso di utilizzo di farmaci diversi per evitare il rischio di formazione di precipitati; il lavaggio con soluzione eparinata deve essere preceduto dal lavaggio con sola soluzione fisiologica perché alcune sostanze reagiscono con l'eparina e possono provocare la formazione di precipitati). Durante la rimozione dell'ago, infine, è corretto mantenere una pressione positiva all'interno dalla siringa per evitare il reflusso di sangue all'interno del catetere. Come suggeriscono Ballerini et al., l'esperienza dello staff chirurgico e infermieristico è probabilmente il fattore cruciale nella riduzione dell'incidenza complessiva di complicanze.

\section{BIBLIOGRAFIA}

1. Ballarini, et al. Complications of subcutaneous infusion port in the genral oncology population. Oncology 1999; 56 (2): 97-102.

2. Murga R, et al. Biofilm formation by gram-negative bacteria on central venous catheter connectors: effect of conditioning films in a laboratory model. J Clin Microbiol 2001; 39 (6): 2294-7.

3. Booth SA, et al. Central venous catheterization and fatal cardiac tamponade. Br J Anaesth 2001; 87 (2): 298-302.

4. Rickard NA. Central venous catheter: infection and patient susceptibility. Br J Nurs 2001; 10 (16): 1044.

5. Reeves AR, et al. Recent trends in central venous catheter placement: a comparison of interventional radiology with other specialties. J Vasc Interv Radiol 2001; 12 (10): 1211-4.

6. Rickard NA. Rudecing infections associated with central venous catheters. Br J Nurs 2003; 12 (5): 274-80.

7. Vesely TM. Central venous catheter tip position: a continuing controversy. J Vasc Interv Radiol 2003; 14 (5): 527-34.

8. Girbes AR, et al. Mechanical and infectious complications of central venous catheters. Minerva Anestesiol 2003; 69 (5): 330-2.

9. Reardon PR, et al. A loop technique for the safe, secure and convenient fixation of subclavian central venous catheters to the chest wall. Am J Surg 2003; 185 (6): 536-7.

10. Jacobs BR. Central venous catheter occlusion and thrombosis. Crit Care Clin 2003; 19 (3): 489-514.

11. Carlon R, et al. Secondary migration of a central venous catheter. A case report. Minerva Anestesiol 2003; 69 (12) : 927-31.

12. Schummer W, et al. Chylothorax 


\section{Giornale \\ di Tecniche \\ Nefrologiche \\ \& Dialitiche \\ Anno XVIII n ${ }^{\circ} 4$ \\ (C) Wichtig Editore 2006

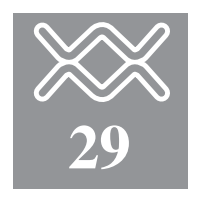

after central venous catheterisation. Considerations to anatomy, differential diagnosis and therapy. Anaesthesist 2003; 52 (10): 919-24.

13. Russel C, et al. Fatal cardiac perforation by central venous catheter dilators : does the lenght matter? Anaesthesia 2003; 58 (12): 1241-2.

14. Laporte $\mathrm{S}$, et al. Thrombosis prophylaxis in cancer patients with a central venous catheter. Arch Intern Med 2004; 164 (4): 459.

15. Salman M, et al. Recurrent laryngeal nerve injury: a complication of central venous catheterization. A case report. Angiology 2004; 55 (3): 345-6.

16. Bona RD. Thrombotic complications of central venous catheter in cancer patients. Semin Thromb Hemost 1999; 25 (2): 147-55.

17. Forauer AR, et al. Histologic changes in the human vein wall adjacent to indwelling central venous catheter. J Vasc Interv Radiol 2003; 14 (9): 1163-8.

18. Nesci M, et al. Vascular catheter positioning and maintenance. Minerva Anestesiol 2002; 68 (5): 492-8.

19. Isaac J, et al. How to avoid fatal complications after central venous catheterization. Br J Anaesth 2002; 88 (1): 147-8.

20. Moureau N, et al. Central venous catheters in home infusion care: outcomes analysis in 50,470 patients. J Vasc Interv Radiol 2002; 13 (10): 1009-16.

21. Life-threatening mediastinal hematoma caused by extravascular infusion through a triple-lumen central venous catheter. Anesth Analg 2004; 99 (1): 31-5.

22. Hartkamp A, et al. Totally implantable venous access devices: evaluation of complications and a prospective comparative study of two different port systems. Neth $\mathrm{J}$ Med 2000; 57 (6): 215-23.

23. O'Grady NP, et al. Guidelines for the prevention of intravascular catheter-related infecttions. Centers for Disease Control and Prevention. MMWR Recomm Rep 2002; 9: 1-29. 